\title{
Influencing Relaxation by a Low Intensity Transcranial Pulsed Magnetic Stimulation Applying the Entrainment Model
}

\author{
Igor Jerman, Primož Dovč, Petra Ratajc \\ Physiological Testing Department, BION, Institute for Bioelectromagnetics and New Biology, Ljubljana, Slovenia \\ Email: igor.jerman@bion.si
}

How to cite this paper: Jerman, I., Dovč, P. and Ratajc, P. (2019) Influencing Relaxation by a Low Intensity Transcranial Pulsed Magnetic Stimulation Applying the Entrainment Model. Open Access Library Journal, 6: e5741.

https://doi.org/10.4236/oalib.1105741

Received: August 29, 2019

Accepted: September 14, 2019

Published: September 17, 2019

Copyright $\odot 2019$ by author(s) and Open Access Library Inc.

This work is licensed under the Creative Commons Attribution International License (CC BY 4.0).

http://creativecommons.org/licenses/by/4.0/

\begin{abstract}
Relaxation is becoming increasingly important in modern stressful times. Besides special psychological practices or consuming pharmaceuticals, one can achieve a more relaxed state also by applying an appropriate magnetic field stimulation of the head (low-intensity rTMS). Following the entrainment model, we did the research using carefully chosen frequencies of modest MF intensity that followed the principles of clinical trials. Besides the situations of blind verum stimulation and the sham one, we tested also the situation that included expectations of the applied stimulation. The testing was done by electrophysiological methods and VAS. The results demonstrated an objective relaxing effect of MF stimulation, where drowsiness was not stimulated. This outcome is much more evident from electrophysiological data than from the VAS ones. From the latter results, we noticed even a slightly negative reaction to expectations.
\end{abstract}

\section{Subject Areas \\ Evolutionary Studies}

\section{Keywords}

Magnetic Field Stimulation, Low Intensity rTMS, PEMF, Relaxation, Electrophysiological Parameters, VAS, Conditions of Clinical Trials

\section{Introduction}

\subsection{State of the Art}

There are many different non-invasive methods of how to achieve a relatively satisfying relaxed state that is increasingly needed in our stressful times. Some of 
them are based on psychological methods, like Shultz's autogenic training including progressive reduction of muscle tension; others on special practices, like meditation or (self) hypnosis. The state of relaxation is a state devoid of tension and anxiety; the deeper the relaxation the lesser tension and anxiety. Relaxation, therefore, brings calmness and diminishes the level of stress hormones like adrenaline and cortisol [1]. The benefits of relaxation are many. Among them, we may enumerate general well-being (higher quality of life), higher power to combat any mental illness issues, a positive influence on somatic health state, a good influence on daily performances including a possible improvement of one's sleep and perhaps overall, the decrease of anxiety [2].

In modern times, however, one may need a deep relaxation, when he/she is not in a position to perform any relaxation practice, while, for instance, driving a car. In such a situation, it would be utterly inadvisable to take medication for deep relaxation in the form of an anxiolytic pill. The latter may have long and undesired negative side effects like drowsiness, as well as slower and less coordinated reactions. A completely different method should be used in such cases, the one that would enable one to simultaneously perform a task without drowsiness and with no mentioned negative side effects. One such possibility is to use pulsed magnetic stimulation (PEMF) in the region of the head.

Magnetic stimulation of the head region is known also under the name repetitive transcranial magnetic stimulation (rTMS). Usually, it uses relatively high intensities of magnetic fields of low frequencies. There are many positive reports concerning this treatment. A comprehensive study published as a review article concludes that although the interpretation of the results is difficult because of different treatment protocols and the lack of a placebo-controlled design in the majority of studies, there is evidence for anxiolytic action of rTMS [3]. It is assumed that the strong MF, capable of eliciting action potentials and applied on the right dorsolateral prefrontal cortex, works inhibitory on the centers that cause anxiety.

Besides reports concerning high-intensity rTMS that have clear physiological effects through magnetic induction of electric fields, some studies claim also biological influences of MF stimulation of low intensities. The latter cannot evoke action potentials; neither do they have thermal effects. Therefore, lacking a general theory of bioelectromagnetics, the mechanism of their bioeffects is still an object of scientific debate. Various models are proposed, some of them with empirical confirmations; nevertheless, no one has been universally recognized (see also [4]). A most plausible assumption is that non-thermal bioeffects should work on the principle of resonance with some physiological and biochemical processes in the body. Most probably, they interfere with the endogenous electromagnetic and dipolar oscillations within cells (for a more comprehensive study see [5]).

When we come to a possibility of influencing relaxation, we may assume the incidental magnetic stimulation will enhance the proportion of brainwaves that 
are in resonance with the frequency of the stimulating magnetic field (MF). This may not necessarily mean only the same frequency matching but may entail also relations between a frequency and its subharmonics or its higher harmonics. The principle, where an exogenous vibration (not necessarily electromagnetic by nature, see also [6]) induces corresponding brainwaves by close frequency matching is known as entrainment. One of the first reports of the entrainment effect elicited by a sinusoidal MF was published by Bell et al. [7]. They found a non-linear enhancing relation between stimulation and the electroencephalogram (EEG) response. Another, more comprehensive study by Cvetković and Čosić [8] comprising four different stimulating frequencies showed that it is possible to influence the alpha and beta waves in stimulated volunteers; however, the effect was not direct under all stimulatory conditions and could be even reverse. The study concluded that an EEG synchronization of driving alpha and beta EEG by corresponding alpha and beta sinusoidal MF stimulation could be applied as treatment(s) of particular neurophysiological abnormalities such as sleep and psychiatric disorders. The stimulating intensities in these two studies span from some tens (the second study) to few hundreds of $\mu \mathrm{T}$ (the first study), while the field itself was produced in Helmholz coils that produce a homogenous MF throughout the head. There are other positive reports regarding the entrainment principle, like the profound study of Thut et al. [8] [9], nevertheless there are also reports that contradict this effect [9] [10] [11] [12]. Cook et al. [13] published a study that tried to find some explanation for these controversies. They found that previous exposure to a pulsed MF sequence determined subjects' responses in the research (see also [14]).

An interesting study was performed by Ghione and colleagues [15] regarding the effects of 90-min exposure of the head to a homogenous $50 \mathrm{~Hz}$ extremely low frequency (ELF) MF at a flux density of 40 or $80 \mu \mathrm{T}$ applied to the head region. They report that the alpha activity after $80 \mu \mathrm{T}$ magnetic treatment almost doubled compared to the sham exposure indicating that, as already said, the entrainment does not necessarily the matching of identical frequencies, but may also be a consequence of subharmonics-higher harmonics matching since approximately two octaves and a half stand between lower-alpha waves and $50 \mathrm{~Hz}$. On the other hand, an influence that may be ascribed to higher harmonics entrainment is reported by Gao et al. [16]. Here, the influence of ELF PEMF with the frequency of $1 \mathrm{~Hz}$ (intensity: $10 \mathrm{mT}$, stimulation duration: $20 \mathrm{~min}$ ) on human brain was conducted. In comparison with the sham exposure group, the EEG power of theta band $(3.5-7.5 \mathrm{~Hz})$ and lower-alpha band $(7.5-10 \mathrm{~Hz})$ from the stimulation group increased significantly after the stimulation. In a direct (same frequency) entrainment case, one would expect the stimulation of delta waves $(0-3.5 \mathrm{~Hz})$, yet approximately the second $(4 \mathrm{~Hz})$ and the third $(8 \mathrm{~Hz})$ octaves involving brainwaves were stimulated. There are also reports concerning the entrainment of theta waves. In a comprehensive study comprising over 100 volunteers, Pelka et al. [17] report a highly significant and positive influence on 
sleep using an overnight MF stimulation at $4 \mathrm{~Hz}$ with an extremely low and varying field intensity (an estimation, not reported in the article: $\sim 10 \mathrm{nT}-1 \mu \mathrm{T}$ ).

\subsection{Background of Present Research, Intentions and Assumptions}

Since there is a controversy concerning the EEG measurements and entrainment expectations, we intended to check what is the effect of a transcranial low-intensity MF stimulation following the entrainment model on various physiological parameters and self-perception of volunteers, in addition to examine only brainwaves. As evident from the previous subchapter for the targeted physiological state, we chose relaxation since it is relatively easy to identify it using electrophysiological and brainwave measurements. Having learned that even subharmonics may have an important influence on brainwaves, we chose two frequencies: a leading one from the alpha region that should be in direct resonance with the targeted brainwave enhancement and a supporting one of a lesser intensity representing a subharmonic from the high delta region (see also Table 1). Our choice for the leading frequency in the middle of the alpha region was based on harmonic, so-called GM-scale, found and published by Meijer and Geesink after a comprehensive review of bioelectromagnetic articles with either stimulatory or inhibitory effects [18]. During MF stimulation of volunteers, our expectation (research assumption) was as follows:

- Brainwaves: Enhancing the proportion of alpha and delta waves during stimulation, sleep should not be induced;

- General electrophysiological parameters: Enhancing the parasympathetic activity, although Ghione et al. [15] found no effect on either blood pressure or heart rate, yet they applied a $50 \mathrm{~Hz} \mathrm{MF}$;

- Self-perception: Identification of a more relaxed state.

In the research we wanted to check also the influence of expectation in combination with MF stimulation on all three above listed groups of parameters, so in addition to the sham-exposed volunteers (control) and the stimulated ones (verum), we examined parameters of volunteers who 1) were stimulated, 2) knew that they were stimulated and 3) were told what should they expect from the stimulation-the informed situation. Our hypothesis here was that the intentionally provoked expectations in the anticipated direction (relaxation) would enhance the influence of MF stimulation.

Although the majority of research in bioelectromagnetics is performed by using a homogenous MF, such field is only very rarely applied in practical situations where an MF stimulation may have some practical or therapeutic use. Namely, on the market devices producing highly non-homogenous MFs are mostly offered, which shaped our decision to apply a highly inhomogeneous field to volunteers. The MF was produced by five different coils arranged around the head (see Figure 1). The measures of a coil were as follows $26.5 \mathrm{~mm}$ (length) $\times 20 \mathrm{~mm}$ (width) $\times 5.1 \mathrm{~mm}$ (thickness). 
Table 1. Parameters of MF stimulation.

\begin{tabular}{llll}
\hline $\begin{array}{l}\text { Frequency } \\
(\mathrm{Hz})\end{array}$ & $\begin{array}{l}\text { Magnetic field } \\
\text { intensity (B) }\end{array}$ & Location & Role \\
\hline 10.12 & $2.5 \mathrm{mT}$ & occiput (1 coil) & $\begin{array}{l}\text { direct entrainment } \\
\text { frequency }\end{array}$ \\
3.375 & $0.25 \mathrm{mT}$ & $\begin{array}{l}\text { occiput (1 coil }), \text { temporal lobes: } \\
\text { left }(2 \text { coils }) \text { and right }(2 \text { coils })\end{array}$ & $\begin{array}{l}\text { supportive subharmonic } \\
\text { frequency }\end{array}$ \\
\hline
\end{tabular}



Figure 1. Example of experimental situation: a volunteer with attached electrodes needed for measurements of physiological parameters and with the stimulating device in place.

\section{Material and Methods}

\subsection{General Data}

\subsubsection{Experimental Situations}

The research was performed in May 2019 in the laboratories of the Institute for Bioelectromagnetics and New Biology (BION Institute) in Ljubljana, Slovenia, EU. In the research, 25 volunteers aged from 29 to 76 years (13 women and 12 men) were subject to three different experimental situations:

1) Double blind sham stimulation (Control situation),

2) Double blind true stimulation (Verum situation),

3) Informed true stimulation as explained in the Introduction (Informed situation).

In the first two situations, the volunteers were treated in conformity with the clinical trials conditions:

- Prospectiveness (general criteria for the effectiveness of the device's activity were determined in advance);

- Placebo effect ruled out (volunteers did not know whether they were stimulated or not);

- Double blind (neither the volunteer nor the research assistant knew whether MF stimulation was applied or not); 
- Randomized (the decisions about sham or true exposure were made randomly).

The volunteers in the Informed situation were intentionally informed about the stimulation; the situation was always applied after the Control and the Verum situations since the volunteers were the same in all three situations.

The volunteers signed an informed consent in which they agreed to cooperate as subjects in this non-invasive scientific research in bioelectromagnetics.

\subsubsection{Stimulating Magnetic Field Characteristics and Positions}

We applied MF stimulation in the region of the head as can be seen in Figure 1 and described in Table 1 . The device generating the MF was manufactured by MDCN Technologies Inc. New York, USA (Omnipemf device) and will be named the device.

During the research, we constantly measured the environmental EM fields and found no significant variation in the frequency range from $5 \mathrm{~Hz}$ to $2 \mathrm{GHz}$ between different days. The geomagnetic field varied from 47,946 to 48,003 nT (data from the nearest INTERMAGNET [19] observatory located in Lonjsko polje, Croatia).

\subsubsection{Physiological Testing and Exposure Procedure}

Each volunteer attended the measurements thrice at the same hour of the day. At the arrival, he/she filled out the questionnaire and completed the visual analog scale (VAS) test (see chapter 2.2 for more detail). After that research assistant placed the device on his/her head in an appropriate position (Figure 1) and attached the electrodes needed for the measurements of physiological parameters. All the preparations before the measurements took about 10 minutes and during this time the device was already stimulating volunteers. This 10 minutes pre-treatment intended was intended to extend the stimulation period to about 40 minutes in total. During the measurements, which lasted for 30 minutes, volunteers sat in a comfortable wooden chair and were instructed to calmly sit until the assistant comes back. The following parameters were measured: brainwave activity (EEG) in four standard frequency spectra (beta waves from $14 \mathrm{~Hz}$ upwards, alpha waves from 8 to $14 \mathrm{~Hz}$, theta waves from 4 to $8 \mathrm{~Hz}$ and delta waves from 0 to $4 \mathrm{~Hz}$ ), skin conductance, heart rate, respiration rate, finger temperature, heart rate variability and thorax expansion depth. The positive electrode for the EEG measurement was placed on the F3 position of the international 10 - 20 EEG system [20] while the negative and ground electrodes were placed on the right and the left earlobe, respectively. After the removal of the electrodes, the volunteer again completed the VAS test and the questionnaire enabling the comparison between before and after for each tested situation.

With every volunteer, we calculated thirty-second medians for the whole measurement period. Based on these data, we calculated aggregated thirty-second medians for all 25 volunteers and used this data to draw graphs for each measured parameter and further statistical analysis. For the sake of analysis and in- 
terpretation of the results, 30 minutes of measurements were split into three parts (each consisting of 20 data points with calculated 30-second median):

- first part-from 0 to 10 minutes,

- second part-from 10 to 20 minutes,

- third part-from 20 to 30 minutes.

For calculating statistically significant differences between any of the three experimental situations, we used the Friedman test. With a post-hoc test (Wilcoxon Signed-Rank test) we determined which comparison demonstrated these differences. The outcome of the results for all tests was corrected by the Holm-Bonferroni correction for multiple comparisons [21].

\subsection{The Visual Analogue Scale (VAS)}

The Visual Analogue Scale (VAS) has been in use for the measurement of subjective parameters that are impossible to measure by any physical method, like pain, anxiety, quality of sleep, etc. since the 1920s [22]. Today it can be used independently or as a supplement to other measurements and may be used even in pharmacology [23]. The scale consists of a line that is usually $100 \mathrm{~mm}$ in length. On each side, there is an anchor descriptor such as very unrelaxed-very relaxed. The volunteer makes a mark on this (normally and in our case horizontal) line, reflecting subjective perception. Afterward, the distance from the left endpoint to the mark is measured in $\mathrm{mm}$. In our case, the VAS line was displayed on the computer screen and the volunteer had to mark the position between the descriptors by using a mouse and clicking the determined point. VAS measurements enable a consequent statistical analysis of data as in any technical device-based measurement systems.

We used the VAS scale for evaluation of the subjective perception of five different parameters: relaxation, energy level, tension, concentration, and fatigue/tiredness. VAS scale was included in online survey volunteers completed before and after each measurement. The online open-source application for web surveys [24] determined the marked position as a distance from the left point in millimeters (range 0 - 100), which was considered as the VAS score. We compared VAS scores of each volunteer before and after individual measurements and also between different measurements (situations): control, verum and informed. Measurements were carried out in a double-blind and randomized fashion, following the same conditions for clinical testing as described in Section 2.1.1.

Besides VAS testing, a question as to when a volunteer expected to be under the true stimulation as well as when they felt better was asked after the second testing (Table 2).

\section{Results}

\subsection{Physiological Testing}

Results concerning electrophysiological measurements demonstrate statistically 
significant differences between three experimental situations for the following parameters: skin conductance, respiration rate, heart rate, the proportion of beta, alpha, theta, and delta waves and thorax expansion depth (Table 3, Friedman test). Skin conductance, heart rate and the proportion of beta and delta waves demonstrated differences in all three parts of measurements.

A post-hoc test (see Table 4, Wilcoxon signed-rank test) reveals that the vast majority of the statistically significant differences came from comparisons between either Verum or Informed situations on one side and the Control on the other. This implies that the Verum and the Informed situations were much more similar to each other than to the Control. This meets our expectations since MF stimulation was applied in both situations.

Table 2. Questions and anchor descriptors for evaluation of five different parameters.

\begin{tabular}{|c|c|c|}
\hline Parameter & Question & Anchor descriptors \\
\hline Relaxation & \multicolumn{2}{|c|}{ How relaxed do you feel at the moment? Very unrelaxed-very relaxed } \\
\hline Energy & $\begin{array}{l}\text { How full of energy do you feel at the } \\
\text { moment? }\end{array}$ & Complete lack of energy_full of energy \\
\hline Concentrati & \multicolumn{2}{|c|}{ How focused do you feel at the moment? Very unfocused-very focused } \\
\hline $\begin{array}{l}\text { Fatigue/ } \\
\text { tiredness }\end{array}$ & How tired do you feel at the moment? & Not tired-very tired \\
\hline Tension & How tense do you feel at the moment? & Very tense-not tense \\
\hline
\end{tabular}

Table 3. p-values of the Friedman test based on 30-second medians for each parameter during the three parts of measurements. Holm-Bonferroni correction for multiple comparisons is applied to the $\mathrm{p}$-values in the table. Bold values denote statistically significant differences between at least two of the three different experimental situations $(p<0.05)$, values with regular black font represent statistically marginally significant differences between at least two of the three different experimental situations $(\mathrm{p}<0.1)$. Marks: $\mathrm{SC}$ - skin conductance, $\mathrm{RR}$ - respiration rate, HR-heart rate, TMP — finger temperature, beta, alpha, theta, and delta - the proportion of beta, alpha, theta and delta spectrum of brain activity, HRV—-heart rate variability, TED—-thorax expansion depth.

\begin{tabular}{cccc}
\hline & $0-10 \mathrm{~min}$ & $10-20 \mathrm{~min}$ & $20-30 \mathrm{~min}$ \\
\hline SC & $\mathbf{0 . 0 0 0}$ & $\mathbf{0 . 0 0 0}$ & $\mathbf{0 . 0 0 0}$ \\
$\mathrm{RR}$ & 1.000 & $\mathbf{0 . 0 2 4}$ & 1.000 \\
$\mathrm{HR}$ & $\mathbf{0 . 0 0 0}$ & $\mathbf{0 . 0 0 0}$ & $\mathbf{0 . 0 0 0}$ \\
TMP & 1.000 & 1.000 & 0.056 \\
beta & $\mathbf{0 . 0 0 0}$ & $\mathbf{0 . 0 0 0}$ & $\mathbf{0 . 0 0 0}$ \\
alpha & 1.000 & $\mathbf{0 . 0 0 0}$ & 1.000 \\
theta & $\mathbf{0 . 0 2 4}$ & 1.000 & 1.000 \\
delta & $\mathbf{0 . 0 0 3}$ & $\mathbf{0 . 0 0 0}$ & $\mathbf{0 . 0 0 0}$ \\
HRV & 0.949 & 1.000 & 1.000 \\
TED & 0.268 & $\mathbf{0 . 0 0 0}$ & $\mathbf{0 . 0 0 0}$ \\
\hline
\end{tabular}


Table 4. p-values of the post-hoc test (Wilcoxon signed-rank test) based on 30-second medians for each parameter during the three parts of measurements. Holm-Bonferroni correction for multiple comparisons is applied. Values with bold black font represent statistically significant differences between two of the selected situations $(\mathrm{p}<0.05)$, values with regular black font represent statistically marginally significant differences between two of the selected situations ( $\mathrm{p}<0.1$ ). Marks: SC—skin conductance, RR-respiration rate, HR-heart rate, TMP - finger temperature, beta, alpha, theta, and delta-the proportion of beta, alpha, theta, and delta spectrum of brain activity, HRV-heart rate variability, TED—thorax expansion depth.

\begin{tabular}{|c|c|c|c|c|c|c|c|c|c|}
\hline \multirow{3}{*}{$\begin{array}{l}\text { comparison } \\
\text { pair }\end{array}$} & \multicolumn{3}{|c|}{$0-10 \mathrm{~min}$} & \multicolumn{3}{|c|}{$10-20 \mathrm{~min}$} & \multicolumn{3}{|c|}{$20-30 \mathrm{~min}$} \\
\hline & Verum & Informed & Verum & Verum & Informed & Verum & Verum I & Informed & Verum \\
\hline & Control & Control & Informed & Control & Control & Informed & Control & Control & Informed \\
\hline SC & 0.008 & 0.017 & 1.000 & 0.008 & 0.128 & 0.008 & 0.008 & 1.000 & 0.008 \\
\hline $\mathrm{RR}$ & 1.000 & 1.000 & 1.000 & 0.227 & 1.000 & 0.070 & 1.000 & 1.000 & 1.000 \\
\hline HR & 0.008 & 0.008 & 1.000 & 0.010 & 0.008 & 0.078 & 0.008 & 0.011 & 1.000 \\
\hline TMP & 1.000 & 1.000 & 1.000 & 1.000 & 1.000 & 1.000 & 0.747 & 1.000 & 0.687 \\
\hline beta & 0.008 & 0.008 & 0.631 & 0.008 & 0.008 & 0.036 & 0.008 & 0.008 & 1.000 \\
\hline alfa & 1.000 & 1.000 & 1.000 & 0.009 & 0.008 & 1.000 & 1.000 & 1.000 & 1.000 \\
\hline theta & 0.022 & 1.000 & 0.128 & 0.579 & 1.000 & 1.000 & 1.000 & 1.000 & 1.000 \\
\hline delta & 0.878 & 0.010 & 1.000 & 0.008 & 0.008 & 1.000 & 0.025 & 0.028 & 1.000 \\
\hline HRV & 1.000 & 1.000 & 1.000 & 1.000 & 1.000 & 1.000 & 1.000 & 1.000 & 1.000 \\
\hline TED & 0.246 & 0.227 & 1.000 & 0.008 & 0.747 & 0.530 & 0.022 & 0.032 & 1.000 \\
\hline
\end{tabular}

Skin conductance was lower for the Verum situation than for the Control one during the entire measurements while it was lower only during the first $15 \mathrm{mi}$ nutes for the Informed situation (Figure 2). Lower skin conductance indicates greater relaxation of volunteers [25]. However, skin conductance indicates that expectation reduced influence of MF stimulation for the Informed situation (a slight negative effect). There were also substantial differences in initial values for this parameter. They were just below $8 \mu \mathrm{S}$ for the Verum and the Informed situations while they were around $9.5 \mu \mathrm{S}$ for the Control situation. We assume that the reason for this initial difference is the 10 minutes pre-treatment.

Relative thorax expansion difference was in many ways similar to skin conductance. Again, values for the Verum situation were lower when compared to the Control for most of the measurements (Figure 3). In addition, the expectation for the Informed situation reduced the impact of the device so that values for this situation were much closer to the Control than to the Informed (a negative psychological influence again). Lower thorax expansion difference is correlated with a lower concentration of adrenaline (within physiological concentrations; see [26]), which is one of the stress hormones and stimulator of the sympathetic nervous system. This result is therefore in harmony with skin conductance.

Heart rate was lower for both the Verum and the Informed situations than for the Control during most of the measurements (Figure 4). The biggest difference 
between the Control and both the Verum and the Informed situations was at the beginning of the measurements and it gradually dwindled towards the end of measurements. It seems that heart rate, similarly to skin conductance, responded to the applied stimulation during pre-treatment in less than 10 minutes. Lower heart rate indicates a greater calmness and relaxation, which is again in harmony with both previously discussed parameters: skin conductance and the thorax expansion difference.

The proportion of alpha waves demonstrated statistically significant differences from the Control situation for both the Verum and the Informed situations only in the second part of the measurements (Figure 5) but shows the same basic trend in all three parts. This is different from skin conductance, thorax expansion difference and heart rate mentioned above, where differences were

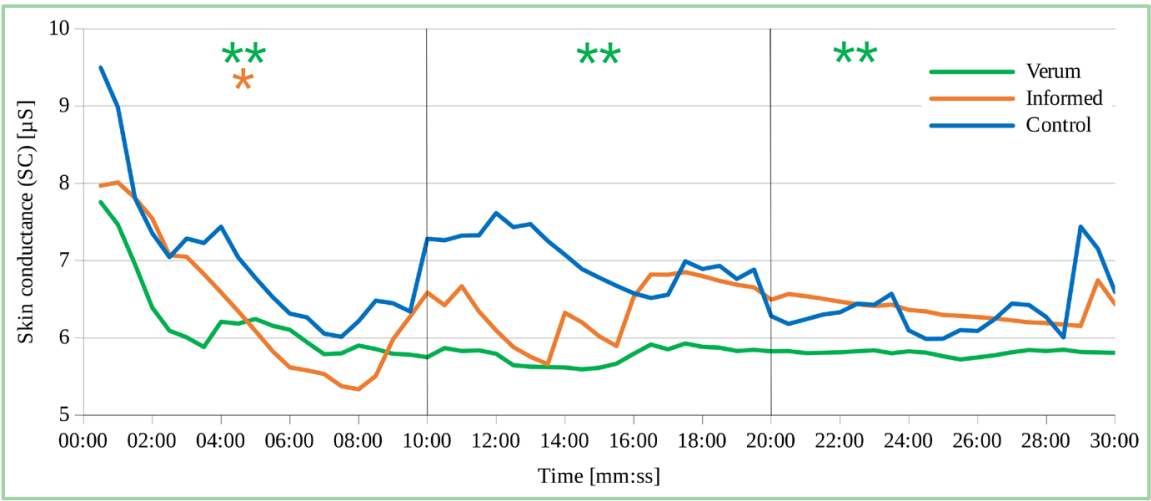

Figure 2. Skin conductance (SC) from twenty-five volunteers (Verum, Informed and Control situations). Asterisks denote statistically significant differences $\left({ }^{*} \mathrm{p}<0.05\right.$; ${ }^{* *} \mathrm{p}<$ 0.01 ) in three parts of measurements separated by vertical lines. Green asterisks: differences between Verum and Control, orange asterisks: differences between Informed and Control. Statistical differences for comparison between Verum and Informed situations are not shown.

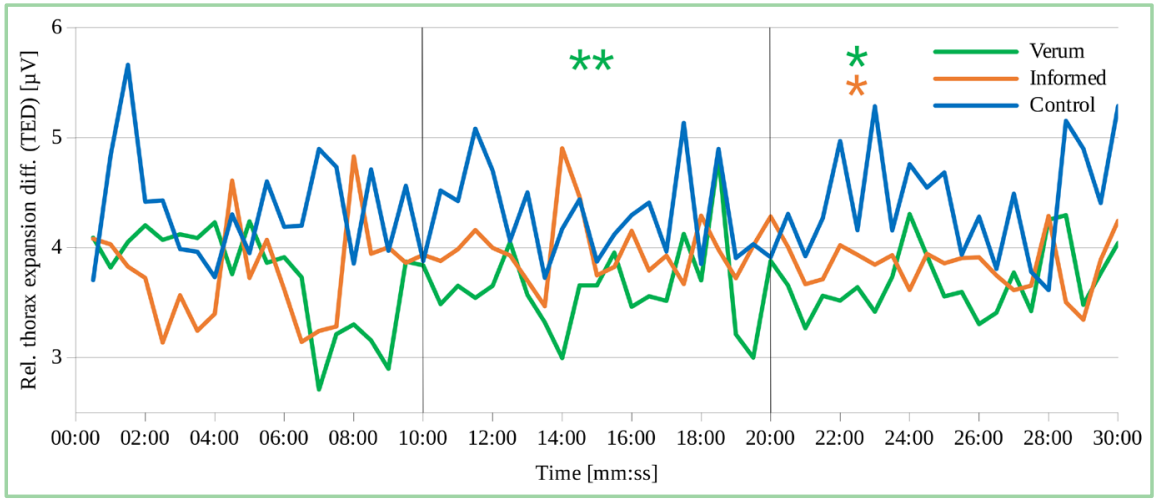

Figure 3. Relative thorax expansion difference (TED) from twenty-five volunteers (Verum, Informed and Control situations). Asterisks denote statistically significant differences $\left({ }^{*} \mathrm{p}<0.05 ;{ }^{* *} \mathrm{p}<0.01\right)$ in three parts of measurements separated by vertical lines. Green asterisks: differences between Verum and Control, orange asterisks: differences between Informed and Control. Statistical differences for comparison between Verum and Informed situations are not shown. 


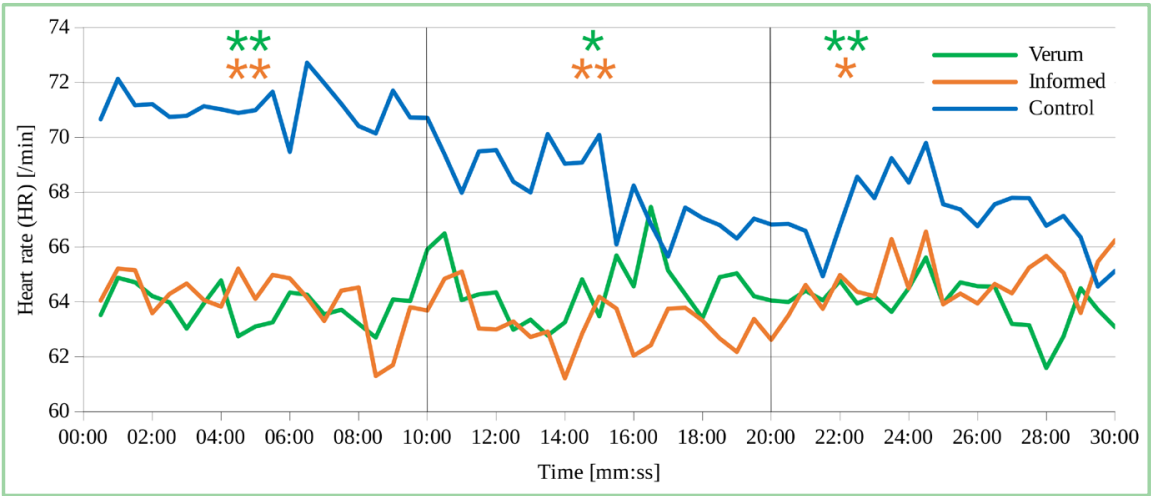

Figure 4. Heart rate (HR) from twenty-five volunteers (Verum, Informed and Control situations). Asterisks denote statistically significant differences $\left({ }^{*} \mathrm{p}<0.05 ;{ }^{* *} \mathrm{p}<0.01\right)$ in three parts of measurements separated by vertical lines. Green asterisks: differences between Verum and Control, orange asterisks: differences between Informed and Control. Statistical differences for comparison between Verum and Informed situations are not shown.

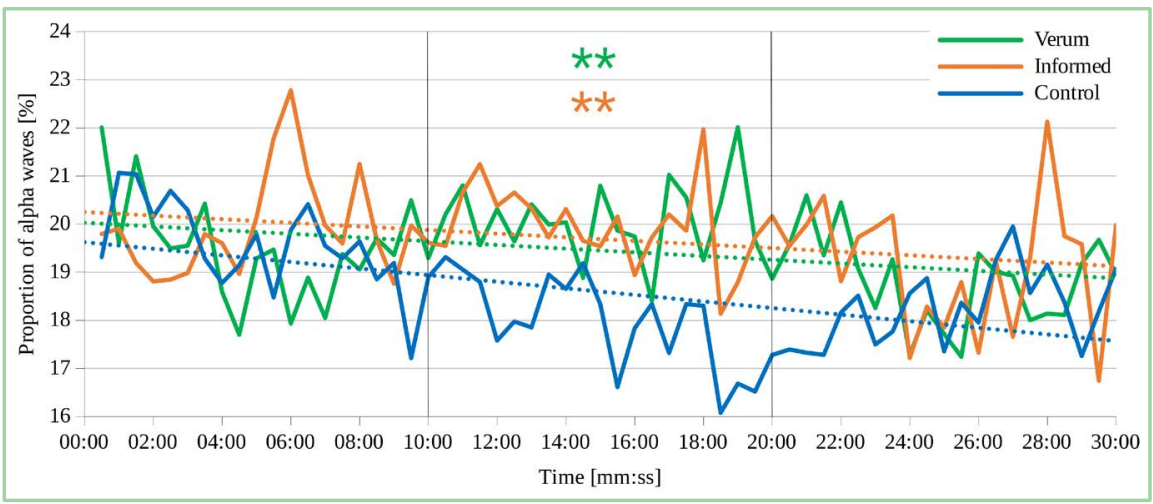

Figure 5. Proportion of alpha waves from twenty-five volunteers (Verum, Informed and Control situations). Asterisks denote statistically significant differences $\left({ }^{* *} p<0.01\right)$ in three parts of measurements separated by vertical lines. Green asterisks: differences between Verum and Control, orange asterisks: differences between Informed and Control. Statistical differences for comparison between Verum and Informed situations are not shown.

more long-lived. Higher alpha waves for the Verum and the Informed situations indicate slightly increased relaxation. Expectation does not seem to have any effect on brainwaves for the alpha spectra as both the Verum and Informed situations followed very similar courses.

Opposite to the proportion of alpha waves, the beta ones demonstrated significant differences from the Control with both the Verum and the Informed situations during the whole length of measurements (Figure 6). Expectations in the Informed situation partially reduced the effect of MF stimulation, especially in the second part of the measurements (slight negative effect). Higher beta waves for both the Verum and the Informed situations indicate a more awakened state despite a higher relaxation. This demonstrates that the stimulation does not induce sleepiness despite inducing relaxation, suggesting for instance that a driver 
can use it while on the road.

Opposite to the proportion of beta waves, the theta ones demonstrated significant differences between the Verum and the Control situation only in the first part of measurements (Figure 7). Once again, the expectation of relaxation reduced the effect of MF stimulation (slight negative effect). Except for the Verum situation in the first part of measurements, the proportion of delta waves demonstrated significant differences from the Control situation with both the Verum and the Informed situations during the whole measurement (Figure 8). Once again, both situations with real stimulation were very close together. The proportion of delta waves and partially the theta ones were lower for both the Verum and the Informed situations indicating that the stimulation reduces sleepiness (Table 5).

\subsection{The Visual Analogue Scale (VAS) Results}

After surveying all VAS measurements, we estimated that only the scores from 20 volunteers were suitable for analysis. Results from five volunteers were excluded due to high initial VAS scores, which did not allow a reliable evaluation of changes during the treatments. Compared to their initial state, volunteers felt more relaxed, energized and focused, and less fatigued and tense after the measurements within any test situations (Figure 9 and Figure 10). A lower increase of energy feeling after the Verum situation was the only statistically significant change of measured parameters compared to the Control situation (paired t-test, see Table 6). However, after the Holm-Bonferroni correction, the p-value of the energy parameter increases to 0.09 .

Table 5. Overview of the mean values for all measured parameters with \pm standard error $(\mathrm{N}=20)$ for all three test situations. Marks: SC—skin conductance, RR—respiration rate, HR — heart rate, TMP — finger temperature, beta, alpha, theta, and delta—the proportion of beta, alpha, theta and delta spectrum of brain activity, HRV—heart rate variability, TED—thorax expansion depth.

\begin{tabular}{|c|c|c|c|c|c|c|c|c|c|}
\hline & & $0-10 \mathrm{~min}$ & & & $10-20 \mathrm{~min}$ & & & $20-30 \mathrm{~min}$ & \\
\hline & Verum & Informed & Control & Verum & Informed & Control & Verum & Informed & Control \\
\hline SC & $16.732 \pm 0.145$ & $14.251 \pm 0.372$ & $20.63 \pm 0.217$ & $15.129 \pm 0.233$ & $14.528 \pm 0.26$ & $20.131 \pm 0.086$ & $14.704 \pm 0.153$ & $12.157 \pm 0.163$ & $20.175 \pm 0.186$ \\
\hline $\mathrm{RR}$ & $17.688 \pm 0.108$ & $17.239 \pm 0.121$ & $17.552 \pm 0.155$ & $17.098 \pm 0.094$ & $16.511 \pm 0.172$ & $17.183 \pm 0.121$ & $16.615 \pm 0.121$ & $16.176 \pm 0.175$ & $16.704 \pm 0.173$ \\
\hline HR & $72.012 \pm 0.186$ & $70.778 \pm 0.296$ & $73.13 \pm 0.248$ & $71.691 \pm 0.176$ & $71.668 \pm 0.277$ & $73.639 \pm 0.317$ & $70.263 \pm 0.228$ & $71.39 \pm 0.314$ & $72.916 \pm 0.342$ \\
\hline TMP & $34.781 \pm 0.084$ & $34.959 \pm 0.041$ & $34.447 \pm 0.046$ & $35.03 \pm 0.031$ & $35.078 \pm 0.018$ & $34.583 \pm 0.032$ & $34.851 \pm 0.02$ & $35.035 \pm 0.022$ & $34.735 \pm 0.019$ \\
\hline beta & $25.607 \pm 0.326$ & $25.644 \pm 0.324$ & $25.585 \pm 0.324$ & $26.797 \pm 0.344$ & $26.74 \pm 0.343$ & $27.01 \pm 0.357$ & $25.396 \pm 0.587$ & $25.314 \pm 0.565$ & $25.316 \pm 0.57$ \\
\hline alpha & $21.267 \pm 0.306$ & $21.306 \pm 0.303$ & $21.397 \pm 0.295$ & $20.694 \pm 0.271$ & $20.804 \pm 0.275$ & $20.925 \pm 0.245$ & $19.53 \pm 0.25$ & $19.583 \pm 0.25$ & $19.495 \pm 0.219$ \\
\hline theta & $23.052 \pm 0.149$ & $23.07 \pm 0.15$ & $23.184 \pm 0.15$ & $23.283 \pm 0.173$ & $23.304 \pm 0.173$ & $23.262 \pm 0.176$ & $24.435 \pm 0.256$ & $24.489 \pm 0.242$ & $24.432 \pm 0.24$ \\
\hline delta & $30.073 \pm 0.321$ & $29.98 \pm 0.31$ & $29.834 \pm 0.297$ & $29.226 \pm 0.344$ & $29.152 \pm 0.345$ & $28.802 \pm 0.254$ & $30.639 \pm 0.346$ & $30.614 \pm 0.339$ & $30.756 \pm 0.313$ \\
\hline HRV & $10.808 \pm 0.436$ & $10.844 \pm 0.348$ & $11.57 \pm 0.56$ & $12.889 \pm 0.373$ & $12.42 \pm 0.372$ & $11.826 \pm 0.4$ & $12.594 \pm 0.68$ & $12.075 \pm 0.499$ & $13.431 \pm 0.488$ \\
\hline TED & $2.678 \pm 0.043$ & $2.51 \pm 0.045$ & $3.681 \pm 0.108$ & $2.828 \pm 0.08$ & $2.797 \pm 0.124$ & $3.33 \pm 0.139$ & $3.05 \pm 0.109$ & $2.92 \pm 0.157$ & $2.919 \pm 0.104$ \\
\hline
\end{tabular}


Table 6. Comparison of changes in mean VAS scores (values after-before) between Control and Verum situations $(\mathrm{N}=20$, paired t-test). Asterisks denote statistically significant differences $(\mathrm{p}<0.05)$.

\begin{tabular}{ccc}
\hline VAS parameter & $\begin{array}{c}\text { p-value } \\
\text { (paired t-test) }\end{array}$ & $\begin{array}{c}\text { p-value corrected } \\
\text { after }\end{array}$ \\
Relaxation & 0.770 & 1.000 \\
$\begin{array}{c}\text { Energy } \\
\text { level }\end{array}$ & $0.018^{*}$ & 0.090 \\
Concentration & 0.598 & 1.000 \\
Fatigue/tiredness & 0.795 & 1.000 \\
Tension & 0.434 & 1.000 \\
\hline
\end{tabular}

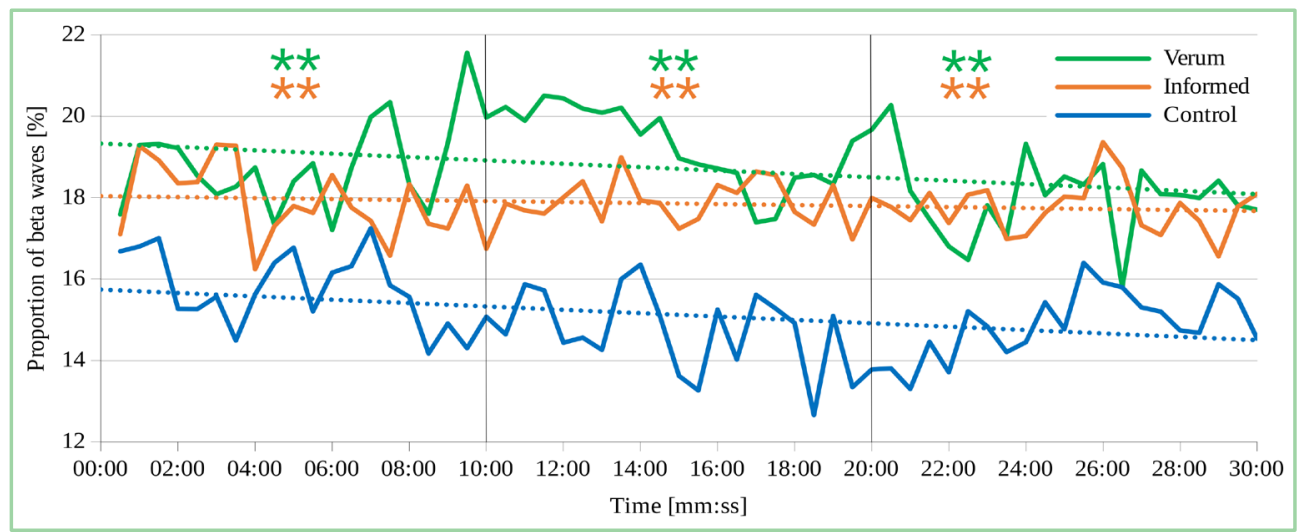

Figure 6. Proportion of beta waves from twenty-five volunteers (Verum, Informed and Control situations). Asterisks denote statistically significant differences $\left({ }^{* *} p<0.01\right)$ in three parts of measurements separated by vertical lines. Green asterisks: differences between Verum and Control, orange asterisks: differences between Informed and Control. Statistical differences for comparison between Verum and Informed situations are not shown.

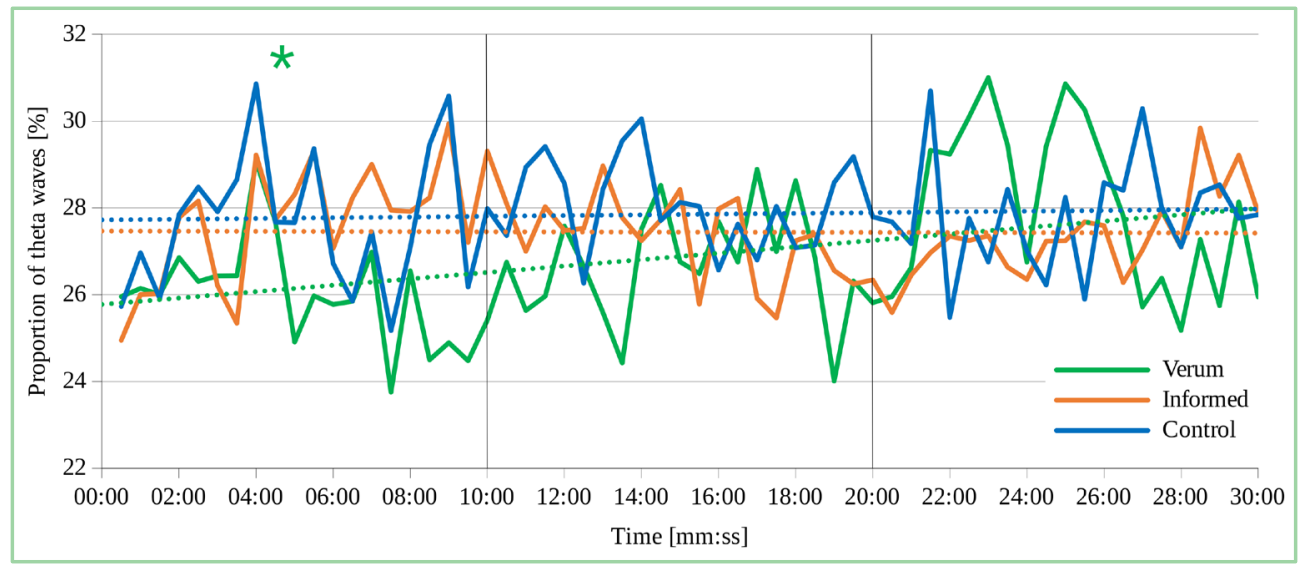

Figure 7. Proportion of theta waves from twenty-five volunteers (Verum, Informed and Control situations). Asterisks denote statistically significant differences $\left({ }^{*} p<0.05\right)$ in three parts of measurements separated by vertical lines. Green asterisk: the difference between Verum and Control. Statistical differences for comparison between Verum and Informed situations are not shown. 


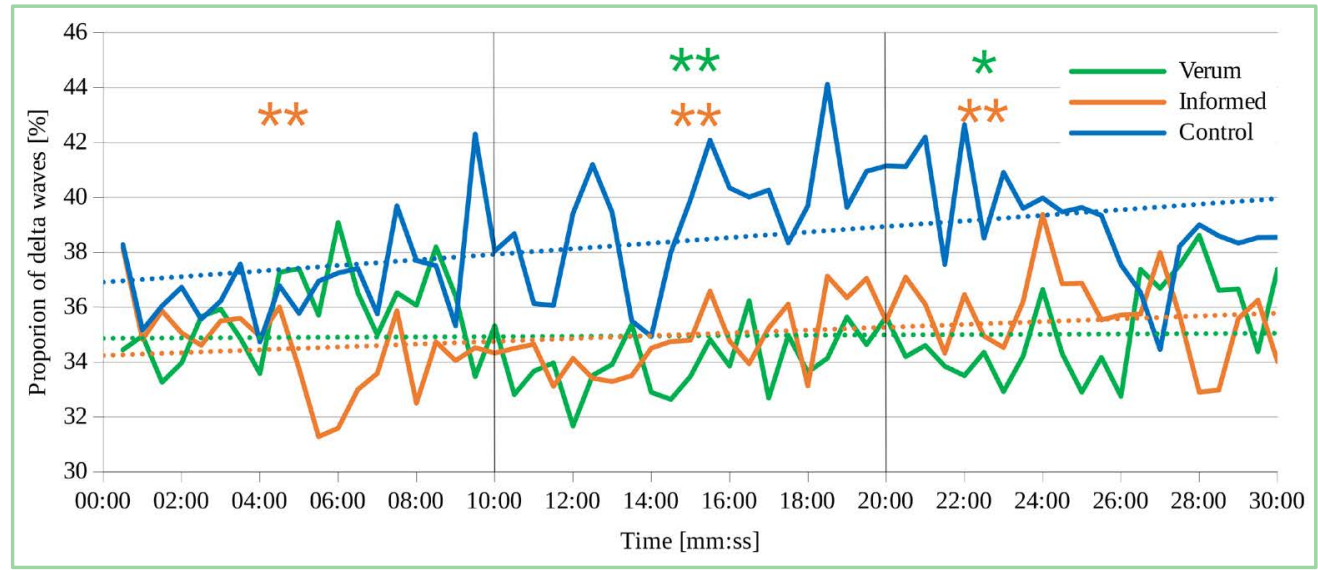

Figure 8. Proportion of delta waves from twenty-five volunteers (Verum, Informed and Control situations). Asterisks denote statistically significant differences $\left({ }^{\star} \mathrm{p}<0.05 ;{ }^{* *} \mathrm{p}<0.01\right)$ in three parts of measurements separated by vertical lines. Green asterisks: differences between Verum and Control, orange asterisks: differences between Informed and Control. Statistical differences for comparison between Verum and Informed situations are not shown.

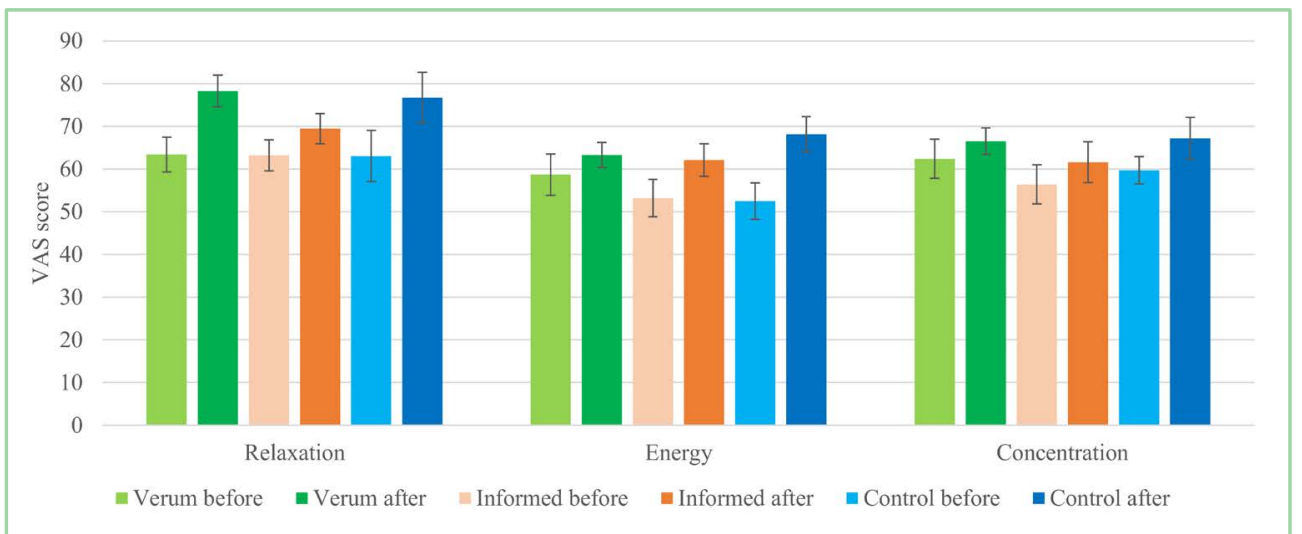

Figure 9. VAS scores of relaxation, energy and concentration before and after the measurements (see the legend below graph bars) with Control, Verum and Informed. Shown are mean values \pm standard error $(\mathrm{N}=20)$.

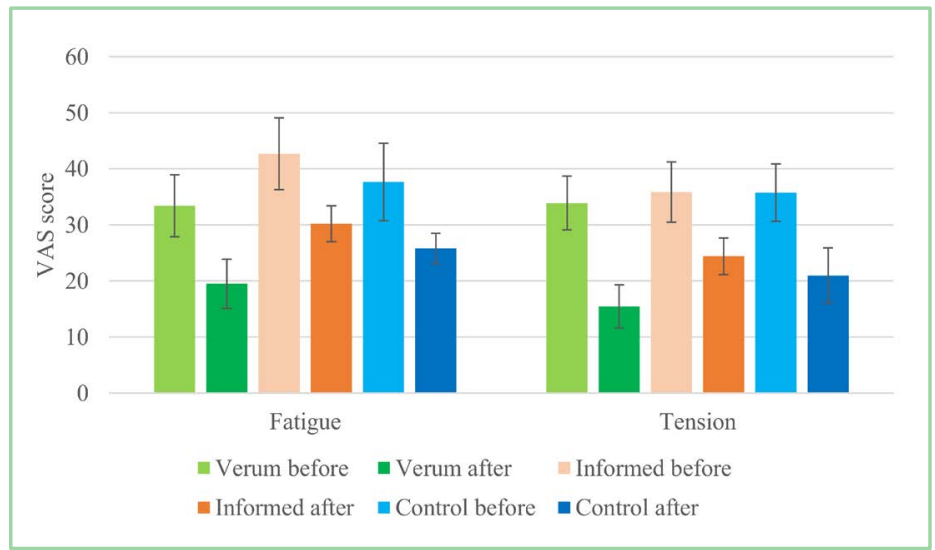

Figure 10. VAS scores of fatigue and tension parameters before and after the measurements (see the legend below graph bars) with Control, Verum and Informed. Shown are mean values \pm standard error $(\mathrm{N}=20)$. 
After completed Control and Verum situations, volunteers were asked to evaluate which measurements made them feel more relaxed. As the research was double-blind and randomized, the possible answers were (A) today's measurement made me feel more relaxed, (B) the previous measurement made me feel more relaxed, (C) both were equally relaxing, (D) I haven't noticed any differences. Results showed that $56 \%$ of volunteers felt more relaxed after the Verum, and $28 \%$ after the Control situation (Figure 11).

\section{Discussion and Conclusion}

\subsection{Interpretation of Results}

If we take the results of physiological measurements first, we may observe some discrepancies between the parameters concerning the working of the autonomic nervous system and brainwaves. Namely, while the parameters SC, HR, and TED for Verum and Informed situations demonstrate that the physiological balance inclines towards parasympathetic stimulation, which conforms to relaxation, the beta waves demonstrate a rise of the median amplitude, which indicates a more attentive state. A significant rise of beta waves during stimulation together with enhancing the alpha waves (at least in the middle period) is a surprise that demands further consideration and research. Enhancing the attentive state is corroborated also by a diminution of theta and delta waves, significantly with the Verum situation. And as already addressed, it is interesting that at least in the middle period (10 - $20 \mathrm{~min}$ ) the median power of alpha waves also increased.

These apparently contradictory results may be interpreted in the sense that the deep effect on the vegetative nervous system of the MF stimulation exhibits the expected calming or anti-stress effect in conformity with the entrainment model expectation. However, it did not convey more sleepiness, but even more vigilance and diminished tendency to fall into sleep. Thinking in terms of usability of such stimulation, it seems it could be very applicable to situations that demand vigilance (like driving a car) and may at the same time tend to provoke stress.

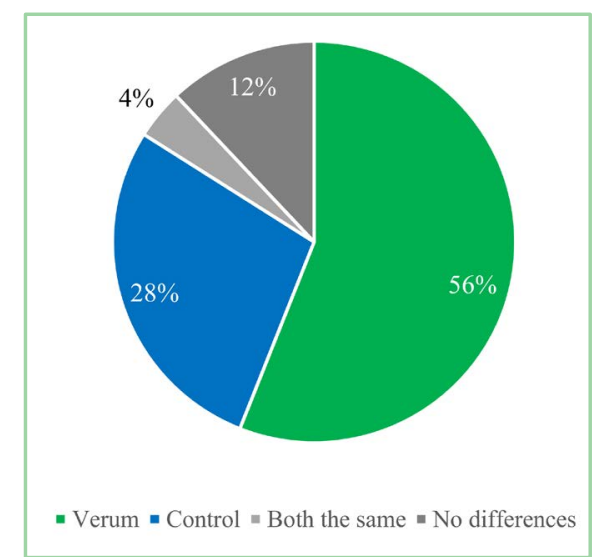

Figure 11. Volunteers' judgment of the more relaxing situation $(\mathrm{N}=25$ volunteers $)$ after concluding both double-blind tests. 
From the results we may also see that all statistically significant results of $\mathrm{Ve}$ rum and Informed situations are of the same sign and very similar; the differences (vs. Control) with the Verum situation tend to be a little more pronounced than with the Informed one. This indicates that acquainting volunteers with the expected influence did not enhance the physiological response, but worked even slightly inhibitory, like a nocebo, which may be seen especially in the results of skin conductance. This difference, small as it is, again corroborates the objective influence of the stimulation regime.

Regarding the VAS testing results, they are not prominent, though there was a tendency to feel a lesser energy level after the Verum stimulation vs. Control. We interpret this in the sense that the volunteers associated a more relaxed and less tense state as the one of a lower energy level, in conformity with the perception of a higher energy level when the concentration of adrenaline in the blood increases. Also worthy of consideration is the direct response of volunteers that in the majority correctly distinguished the difference between Verum and Control and the fact that a significant proportion of volunteers recognized the Verum stimulation as more pleasant.

The discrepancy between the results of electrophysiological and VAS measurement may reflect two circumstances. The first concerns our long experience that there is a huge hiatus between registrations of deep subconscious levels (autonomous nervous system) and consciousness. Accordingly, subtle influences that are registered by the autonomic nervous system remain largely unregistered on the level of normal waking consciousness; and VAS concerns the latter. The second tackles VAS itself. Namely, it is normally used in situations, where it is very clear, what the measured parameter means, like for instance pain. It has a special meaning for volunteers since it tackles one of their most prominent problems. In our testing, the volunteers did not have a general issue to be cured or ameliorated. On average, they did not have a clear relation towards any of the five parameters and therefore they might have not succeeded to connect subtle differences that may have appeared in their minds to VAS questions. In the future, researches concerning MF stimulation, one should try to find parameters that may have a clearer meaning for an average volunteer.

A slight negative (nocebo) effect observed with some physiological and VAS parameters may be interpreted as a psychological reaction of volunteers to expectations. According to our assumption it tackles the psychological level of volunteers and tells that expectancy of relaxation can hinder objective stimulatory effects of the pulsed MF.

\subsection{Conclusions}

- A much higher number of statistical differences (after Holm-Bonferroni corrections) between either Verum and Control or Informed and Control in comparison to Verum vs. Informed strongly confirm the objective effect of the stimulating field of the device. 
- The expectation that the autonomous nervous system will be stimulated in the parasympathetic direction was also confirmed (especially SC and HR parameters).

- The expected entrainment in alpha waves was only partially confirmed since it showed itself only in the middle period of both situations involving MF stimulation.

- The hypothesis that the passage to sleep will not be stimulated was also confirmed.

- Relatively small differences between Verum and Informed situations speak that

$\diamond$ they felt better after it,

$\diamond$ the stimulating magnetic field effect on human physiology is much stronger than psychological influences based on expectancies.

- It seems that in this research situation expecting relaxation brings a measure of stress, which was reflected in a frequent lowering of Informed values in comparison to the Verum ones.

- From VAS and queries after testing, we may conclude that

$\diamond$ the volunteers predominantly recognized the true stimulation and

$\diamond$ they felt better after it.

- Much stronger results (statistically regarded and in the magnitude of differences) in the physiological part of the research than VAS and queries confirm our already known experiences that physiology is a much better detector of various fields' influences than psychology (awareness).

\subsection{Guidelines for Future Research}

The future research on this line should include

- duration of the physiological changes after the end of stimulation,

- some other combinations of frequencies regarding optimization of entrainment,

- inclusion of a positive control into research,

- research of pure placebo (no stimulation, but informed as if stimulated) influence,

- optimization of VAS testing,

- researching the best intensity windows for stimulating relaxation in the direction of lower intensities.

\section{Acknowledgements}

This work was supported by MDCN Technologies Inc. New York, USA. and MDCN Tech Ltd. Slovenia, EU. We would like to thank Vesna Periček Krapež and Mateja Senica for reviewing and correcting the manuscript.

\section{Conflicts of Interest}

The authors declare no conflicts of interest regarding the publication of this paper. 


\section{References}

[1] Cruess, D.G., Antoni, M.H., Kumar, M. and Schneiderman, N. (2000) Reductions in Salivary Cortisol Are Associated with Mood Improvement during Relaxation Training among HIV-Seropositive Men. Journal of Behavioral Medicine, 23, 107-122. https://doi.org/10.1023/A:1005419917023

[2] Guzicki, J.A., Coates, T.J. and Goodwin, D.L. (1980) Reductions in Anxiety and Improvements in Teaching Associated with Cue-Controlled Relaxation Training. Journal of School Psychology, 18, 17-24. https://doi.org/10.1016/0022-4405(80)90044-8

[3] Zwanzger, P., Fallgatter, A.J., Zavorotnyy, M. and Padberg, F. (2009) Anxiolytic Effects of Transcranial Magnetic Stimulation-An Alternative Treatment Option in Anxiety Disorders? Journal of Neural Transmission, 116, 767-775. https://doi.org/10.1007/s00702-008-0162-0

[4] Markov, M.S. (2007) Magnetic Field Therapy: A Review. Electromagnetic Biology and Medicine, 26, 1-23. https://doi.org/10.1080/15368370600925342

[5] Meijer, D.K.F. and Geesink, J.H. (2019) Life and Consciousness are Guided by a Semi-Harmonic EM Background Field. NeuroQuantology, 17, 37-44.

https://doi.org/10.14704/nq.2019.17.4.2074

[6] Roberts, B.M., Clarke, A., Addante, R.J. and Ranganath, C. (2018) Entrainment Enhances Theta Oscillations and Improves Episodic Memory. Cognitive Neuroscience, 9, 181-193. https://doi.org/10.1080/17588928.2018.1521386

[7] Bell, G.B., Marino, A.A. and Chesson, A.L. (1994) Frequency-Specific Responses in the Human Brain Caused by Electromagnetic Fields. Journal of the Neurological Sciences, 123, 26-32. https://doi.org/10.1016/0022-510X(94)90199-6

[8] Cvetković, D. and Čosić, I. (2009) Alterations of Human Electroencephalographic Activity Caused by Multiple Extremely Low Frequency Magnetic Field Exposures. Medical \& Biological Engineering \& Computing, 47, 1063-1073. https://doi.org/10.1007/s11517-009-0525-1

[9] Thut, G., Veniero, D., Romei, V., Miniussi, C., Schyns, P. and Gross, J. (2011) Rhythmic TMS Causes Local Entrainment of Natural Oscillatory Signatures. Current Biology, 21, 1176-1185. https://doi.org/10.1016/j.cub.2011.05.049

[10] Thut, G., Schyns, P. and Gross, J. (2011) Entrainment of Perceptually Relevant Brain Oscillations by Non-Invasive Rhythmic Stimulation of the Human Brain. Frontiers in Psychology, 2, 170. https://doi.org/10.3389/fpsyg.2011.00170

[11] Stodilka, R., et al. (2011) Pulsed Magnetic Field Exposure Induces Lasting Changes in Neural Network Dynamics. Neurocomputing, 74, 2164-2175.

https://doi.org/10.1016/j.neucom.2011.01.025

[12] Dufor, T. (2017) Low Intensity rTMS to the Cerebellum: Age Dependent Effects and Mechanisms Underlying Neural Circuit Plasticity. Doctoral Dissertation, Université Pierre et Marie Curie-Paris VI, Paris.

[13] Cook, C.M., Saucier, D.M., Thomas, A.W. and Prato, F.S. (2009) Changes in Human EEG Alpha Activity Following Exposure to Two Different Pulsed Magnetic Field Sequences. Bioelectromagnetics, 30, 9-20. https://doi.org/10.1002/bem.20434

[14] Cook, C.M., Thomas, A.W., Keenliside, L. and Prato, F.S. (2005) Resting EEG Effects during Exposure to a Pulsed ELF Magnetic Field. Bioelectromagnetics, 26, 367-376. https://doi.org/10.1002/bem.20113

[15] Ghione, S., Del Seppia, C., Mezzasalma, L. and Bonfiglio, L. (2005) Effects of $50 \mathrm{~Hz}$ Electromagnetic Fields on Electroencephalographic Alpha Activity, Dental Pain 
Threshold and Cardiovascular Parameters in Humans. Neuroscience Letters, 382, 112-117. https://doi.org/10.1016/j.neulet.2005.02.072

[16] Gao, X., Wang, X., Chen, F., Qi, H., Wang, X., Ming, D. and Zhou, P. (2014) Research on Brain Induced Effect by Extremely Low Frequency Pulsed Magnetic Stimulation. 2014 36th Annual International Conference of the IEEE Engineering in Medicine and Biology Society, Chicago, IL, 26-30 August 2014, 2613-2616.

[17] Pelka, R.B., Jaenicke, C. and Gruenwald, J. (2001) Impulse Magnetic-Field Therapy for Insomnia: A Double-Blind, Placebo-Controlled Study. Advances in Therapy, 18, 174-180. https://doi.org/10.1007/BF02850111

[18] Geesink, J.H. and Meijer, D.K.F. (2017) Electromagnetic Frequency Patterns that Are Crucial for Health and Disease Reveal a Generalized Biophysical Principle: The GM Scale. Quantum Biosystems, 8, 1-16.

[19] INTERMAGNET (2019) International Real-Time Magnetic Observatory Network. http://www.intermagnet.org/data-donnee/dataplot-eng.php?type $=\mathrm{hdz}$

[20] Jasper, H. (1958) Report of the Committee on Methods of Clinical Examination in Electroencephalography. Electroencephalography and Clinical Neurophysiology, 10, 370-375. https://doi.org/10.1016/0013-4694(58)90053-1

[21] Holm, S. (1979) A Simple Sequentially Rejective Multiple Test Procedure. Scandinavian Journal of Statistics, 6, 65-70.

[22] Heller, G.Z., Manuguerra, M. and Chow, R. (2016) How to Analyze the Visual Analogue Scale: Myths, Truths and Clinical Relevance. Scandinavian Journal of Pain, 13, 67-75. https://doi.org/10.1016/j.sjpain.2016.06.012

[23] Wilhelmus, M.M., et al. (2017) Effects of a Single, Oral $60 \mathrm{mg}$ Caffeine Dose on Attention in Healthy Adult Subjects. Journal of Psychopharmacology, 31, 222-232. https://doi.org/10.1177/0269881116668593

[24] 1KA (2019) 1KA (Version 19.06.07) (Software). Faculty of Social Sciences, Ljubljana. https://www.1ka.si

[25] Borchardt, A.R. and Zoccola, P.M. (2018) Recovery from Stress: An Experimental Examination of Focused Attention Meditation in Novices. Journal of Behavioral Medicine, 41, 836-849. https://doi.org/10.1007/s10865-018-9932-9

[26] Nice, L.B., Rock, J.L. and Courtright, R.O. (1914) The Influence of Adrenaline on Respiration. American Journal of Physiology-Legacy Content, 34, 326-331. https://doi.org/10.1152/ajplegacy.1914.34.3.326 